\title{
Lycopene inhibits neointimal hyperplasia through regulating lipid metabolism and suppressing oxidative stress
}

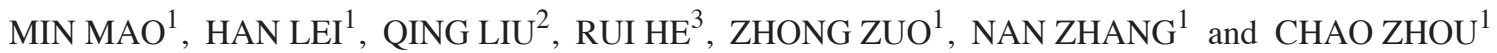 \\ ${ }^{1}$ Department of Cardiology; ${ }^{2}$ Centre for Clinical Research, The First Affiliated Hospital of Chongqing Medical University, \\ Chongqing 400016; ${ }^{3}$ Department of Cardiology, Chongqing Zhongshan Hospital, Chongqing 400013, P.R. China
}

Received December 5, 2013; Accepted March 11, 2014

DOI: $10.3892 / \mathrm{mmr} .2014 .2186$

\begin{abstract}
The present study aimed to investigate the possible mechanism(s) through which lycopene inhibits neointimal hyperplasia in restenosis models. A total of 32 white rabbits were randomly divided into the following four groups: A sham group, a model group, a model group treated with apocynin and a model group treated with lycopene. Immunohistochemistry and transmission electron microscopy (TEM) were used to detect the carotid structures in these groups. Quantitative polymerase chain reaction (qPCR) and western blot analysis were performed to detect the mRNA and protein expression levels of the proteins involved in cell proliferation and oxidative stress, including anti-proliferating cell nuclear antigen, extracellular signal-regulated kinase, nicotinamide adenine dinucleotide phosphate oxidase 1 , hydroxymethyl glutaric acyl coenzyme A reductase, adenosine triphosphate-binding cassette transporter A1 and human neutrophil cytochrome b light chain. Immunohistochemistry and TEM indicated that lycopene treatment significantly reduced the intima/media ratios, the accumulation of lipids and the formation of foam cells in carotid plaques in rabbit restenosis models. Furthermore, lycopene regulated the blood lipid levels and suppressed the oxidative stress in these models. In addition, qPCR and western blot analyses revealed that lycopene significantly decreased the expression levels of cell proliferation-associated proteins, as well as proteins involved in lipid synthesis and transportation. The results suggest that lycopene may regulate lipid metabolism and suppress oxidative stress, and thus, represents a promising therapeutic against neointimal hyperplasia.
\end{abstract}

Correspondence to: Dr Han Lei, Department of Cardiology, The First Affiliated Hospital of Chongqing Medical University, 1 Youyi Road, Yuzhong, Chongqing 400016, P.R. China

E-mail: maominmaomin123@163.com

Key words: lycopene, neointimal hyperplasia, oxidative stress, lipid metabolism

\section{Introduction}

Vascular restenosis is a common complication of percutaneous transluminal coronary angioplasty (PTCA) and stent implantation, which severely compromises the efficacy of treatment. Despite improvements in technology, the incidence of restenosis remains high due to neointimal hyperplasia, which results from mechanical dilating injuries and may be augmented by the presence of the stent (1). Under these circumstances, the supplementation of pharmacological treatments limiting neointimal ingrowth may improve the therapeutic outcomes.

As shown in a previous study, proliferating cell nuclear antigen (PCNA) and extracellular signal-regulated kinase (ERK) $1 / 2$ exhibit important roles in neointimal proliferation. PCNA, which is a nuclear polypeptide synthesized or expressed only in proliferative cells, can be used as an evaluation index of cell proliferation $(2,3)$. ERK, a type of mitogen-activated protein kinase, mainly mediates cell proliferation induced by growth factors and cytokines (4). Reactive oxygen species (ROS) produced by nicotinamide adenine dinucleotide phosphate-oxidase (NADPH oxidase) can promote cell proliferation in the smooth muscle of blood vessels (5). Apocynin is a specific inhibitor of NADPH oxidase, which inhibits neointimal proliferation by inhibiting the expression of NADPH oxidase (6). Thus, the proper use of drugs which can promote or inhibit the secretion of the aforementioned elements may effectively prevent diseases and lycopene is one of them.

Lycopene, a type of carotenoid, is a natural component of tomatoes and tomato products. Previous epidemiological studies have demonstrated an inverse correlation between the lycopene levels in serum and adipose tissues and the incidence of cardiovascular diseases (7-9). Furthermore, lycopene levels in serum and other tissues has been negatively correlated with the thickness of the intima in carotid and aortic plaques $(10,11)$, suggesting that lycopene may inhibit arterial intimal hyperplasia. In the present study, vascular restenosis models were established through carotid balloon injury and a high-fat diet. The effects of lycopene on blood lipids, lipid peroxidation and intimal hyperplasia, as well as the expression levels of proteins involved in cell proliferation, oxidative stress and lipid metabolism, were investigated, and the associated mechanisms were also examined. 


\section{Materials and methods}

Animals. The experimental procedures and animal care were approved by the Experimental Animal Ethics Committee of Chongqing Medical University (Chongqing, China). A total of 32 healthy adult male New Zealand white rabbits, weighing $2.23 \pm 0.18 \mathrm{~kg}$, were included in the present study. The animals were randomly divided into the following four groups ( $n=8 /$ group): A sham group, a model group, a model group treated with apocynin and a model group treated with lycopene. The sham group was exposed to surgery without artery injury, while the other three groups were subjected to artery injury. For drug administration, the sham and model groups were administered a placebo (DSM Nutritional Products, Basel, Switzerland) containing all the ingredients of lycopene or apocynin beadlets with the exception of the lycopene or apocynin. The animals in the lycopene group and the apocynin group were fed with a diet mixed with lycopene (10 $\mathrm{mg} / \mathrm{kg}$ body weight/day) and apocynin $(10 \mathrm{mg} / \mathrm{kg}$ body weight/day), respectively. Lycopene and apocynin were generously donated by DSM Nutritional Products (Basel, Switzerland).

Hematoxylin and eosin $(H \& E)$ staining. A total of $24 \mathrm{~h}$ following fixation with $4 \%$ paraformaldehyde, the arterial samples were dehydrated, embedded in paraffin and then cut into $5 \mu \mathrm{m}$ sections. Standard H\&E staining was performed on these serial sections. Briefly, the slices were stained with $\mathrm{H} \& \mathrm{E}$ for 3 min until the nuclei were blue. They were then subjected to visualization under a light microscope (Axio Imager 2; Carl Zeiss, Oberkochen, Germany). Image Pro Plus 6.0 software (Media Cybernetics, Rockville, MD, USA) was used for image analysis. The thicknesses of the intima and media were measured and the intima/media thickness ratio (IT/MT) was calculated accordingly.

Transmission electron microscopy (TEM). The samples for TEM examination were fixed in $2.5 \%$ glutaraldehyde in $0.1 \mathrm{M}$ sodium phosphate buffer $\left(\mathrm{pH} \mathrm{7.35)}\right.$ for $2 \mathrm{~h}$ at $4^{\circ} \mathrm{C}$. The tissues were successively rinsed in sodium phosphate buffer ( $\mathrm{pH} 7.35$ ) and $2 \%$ osmium tetroxide for $2 \mathrm{~h}$ at room temperature. Following dehydration in gradient acetone series, the tissues were embedded in Epon-Araldite-DDSA and then cut into $60 \mathrm{~nm}$ sections. The sections on the grids were stained with lead citrate for 3 min and then observed on a transmission electron microscope (JEM-2100F; Hitachi, Toyota, Japan).

Blood and vascular sample collection. For sampling, the rabbits were anesthetized and the blood was collected through heart puncture. Plasma and serum were obtained for further analysis. At the end of the study, the left (injured) and right (uninjured) common carotid arteries were removed. Each carotid was cut into two sections, one of which was used for immunohistochemical analysis and the other was used for quantitative polymerase chain reaction (qPCR) and western blot analyses.

Detection of serum lipid levels and lipid peroxidation. Total cholesterol (TC), triglycerides (TG), low-density lipoprotein cholesterol (LDL-C), high-density lipoprotein cholesterol (HDL-C), superoxide dismutase (SOD), total anti-oxidant capacity (T-AOC) and malondialdehyde (MDA) were measured
Table I. Primer sequences for quantitative polymerase chain reaction.

\begin{tabular}{ll}
\hline Primers & \multicolumn{1}{c}{ Sequences } \\
\hline PCNA & F: 5'-GGCTGAAGATAATGCGGACA-3' \\
& R: 5'-CGGTGAGGCGAAAGAGGA-3' \\
ERK1/2 & F: 5'-TATGGGGAAAGTTAGCATTG-3' \\
& R: 5'-GTTACTCGGAGGAGGCTT-3' \\
Nox1 & F: 5'-TTGTTTCTGGTTGTTTGGTTAG-3' \\
& R: 5'-GCTTTGTGCTGTCACCTCATA-3' \\
p22 ${ }^{\text {phox }}$ & F: 5'-TCCTCCTTGCTACCATCCTG-3' \\
& R: 5'-TTCGTTGGCGGGTCGTTG-3' \\
HMG-CoA R & F: 5'-GCAGTCAGTGGGAACTATTTCTG-3' \\
& R: 5'-GCAGTCAGTGGGAACTATTTCTG-3' \\
ABCA1 & F: 5'-AATGATTCGGACATAGACC-3' \\
& R: 5'-TTGACGACTTGCGGGAGT-3' \\
GAPDH & F: 5'-GACATCAAGAAGGTGGTGAAGC-3' \\
& R: 5'-CAGCATCGAAGGTAGAGGAGTG-3'
\end{tabular}

PCNA; anti-proliferating cell nuclear antigen; ERK; extracellular signal-regulated kinase; Nox1, nicotinamide adenine dinucleotide phosphate oxidase 1; HMG-CoA R, hydroxymethyl glutaric acyl coenzyme A reductase; ABCA1, adenosine triphosphate-binding cassette transporter A1; p22 $2^{\text {phox }}$, human neutrophil cytochrome b light chain.

by detection kits (TC kit, cat. no. F002-2; TG kit, cat. no. F001-1; Serum LDL-C kit, cat. no. F004-2; Serum HDL-C kit, cat. no. F003-2; SOD kit, cat. no. A001-1; T-AOC kit, cat. no. A015; MDA kit, cat. no. A003-1, respectively) purchased from the Nanjing Jiancheng Bioengineering Institute (Nanjing, China) according to the manufacturer's instructions. The serum samples were naturally thawed at room temperature. The activities and/or contents of SOD, MDA and T-AOC were assessed by the xanthine oxidase (hydroxylamine) method, a 2-thiobarbituric acid (TBA) assay and a colorimetric test, respectively.

$q P C R$. Total RNA was isolated from the rabbit carotid tissues using the RNAiso Plus kit (Takara, Dalian, China). A total of 500 ng RNA was used as templates for cDNA generation with the RNA RT kit (Takara). PCR was performed using a sequence detection system (CFX96TM Real-Time PCR Detection System; Bio-Rad, Hercules, CA, USA), using Power SYBR Green PCR master mix (Takara). $\beta$-actin served as the reference housekeeping gene. The $2^{-\Delta \Delta \mathrm{Ct}}$ method was applied to obtain the relative expression levels of the target genes. The primer sequences used in the present study are summarized in Table I.

Western blot analysis. The total proteins were extracted and then separated by SDS-PAGE. The proteins were then transferred onto nitrocellulose membranes and the membranes were incubated with anti-PCNA antibody (1:1,000 dilution; LifeSpan BioSciences, Inc., Seattle, WA, USA), anti-ERK 1/2 antibody (1:2,000 dilution; LifeSpan BioSciences, Inc.), anti-phosphorylated (p)-ERK1/2, antibody (1:2,000 dilution; LifeSpan BioSciences, Inc.), anti-nicotinamide adenine dinucleotide phosphate oxidase 1 (Nox1) antibody (1:1,000 dilution; Abcam, Cambridge, UK), anti-human neutrophil cytochrome b light chain (p22 $\left.{ }^{\text {phox }}\right)$ antibody (1:1,000 dilution; Abcam), anti-hydroxymethyl glutaric acyl coenzyme A (HMG-CoA) reductase 

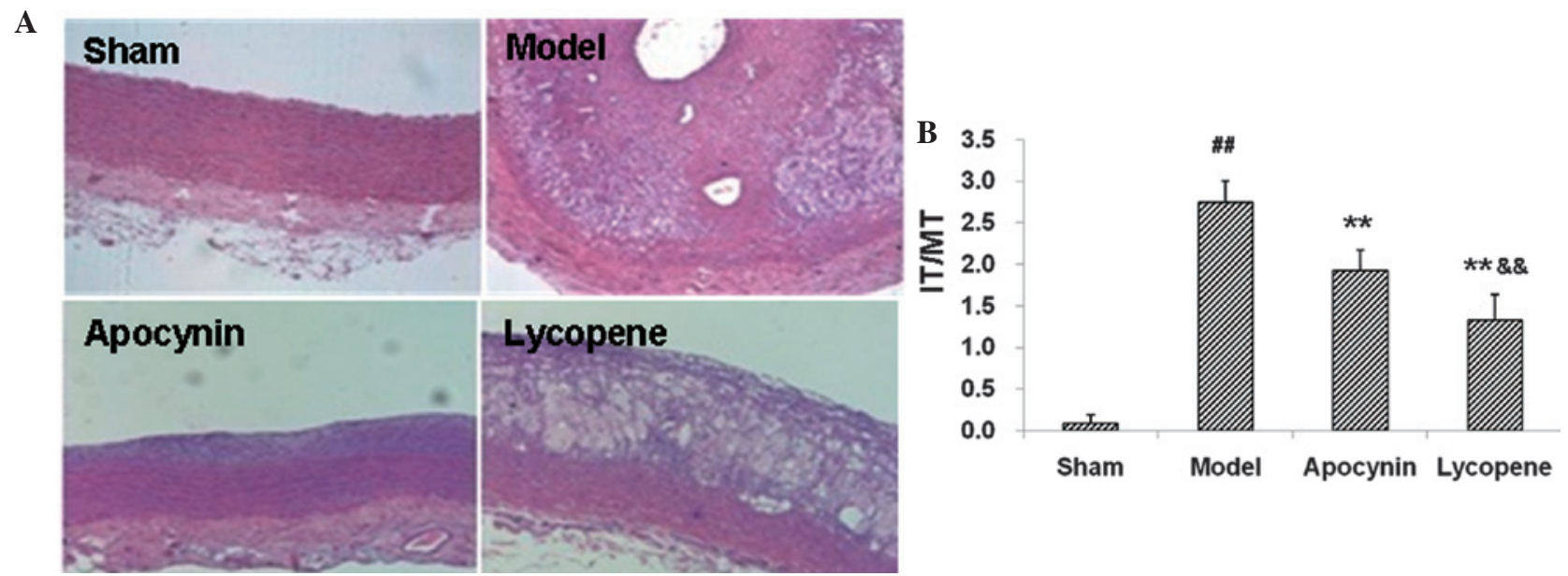

Figure 1. Carotid structures in rabbit restenosis models. (A) Carotid structures in restenosis were detected with hematoxylin and eosin staining (magnification, $\mathrm{x} 200$ ). (B) Statistical analysis of the IT/MT ratios in the restenosis models. ${ }^{\# \#} \mathrm{P}<0.01$, compared with the sham group; ${ }^{* *} \mathrm{P}<0.01$, compared with the model group; \&\& $\mathrm{P}<0.01$, compared with the apocynin group. IT/MT, intima/media thickness ratio.

A

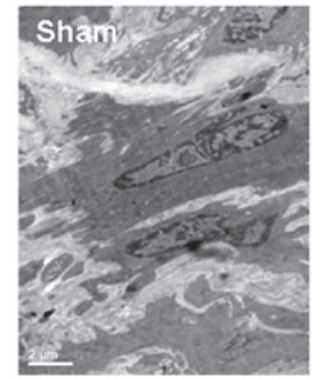

B

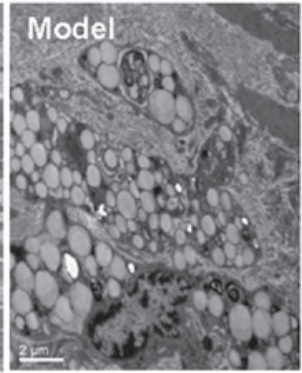

C

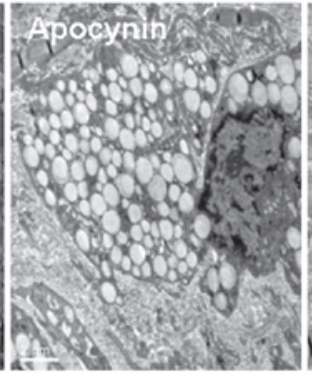

D

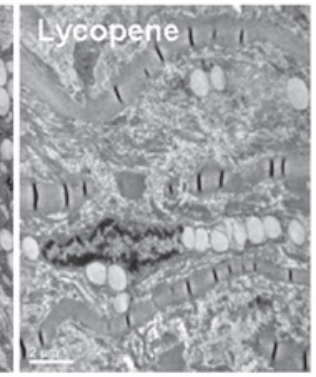

Figure 2. Carotid structures detected with transmission electron microscopy (magnification, $\mathrm{x} 7,000)$. The carotid structures, the formation of the foam cells and the morphologies of smooth muscle cells were visualized with TEM in (A) the sham group, (B) the model group, (C) the apocynin-treated group and (D) the lycopene-treated group.

antibody (1:1,000 dilution; Abcam) and anti-ATP-binding cassette transporter A1 (ABCA1) antibody (1:1,000 dilution; Abcam), respectively, at $4^{\circ} \mathrm{C}$ overnight. The membranes were further incubated with horseradish peroxidase-conjugated goat anti-mouse immunoglobulin G (1:4,000 dilution; ZSGB-BIO, Beijing, China) at room temperature for another $1 \mathrm{~h}$. Following washing, the membranes were analyzed using an enhanced chemiluminescence advanced system (Amersham Biosciences, Piscataway, NJ, USA). Actin was used as a loading control. The protein bands on the membrane were analyzed using a biological image analysis system (Quantity One; Bio-Rad, Richmond, VA, USA) to assess the protein expression levels.

Statistical analysis. Data are presented as the mean \pm standard deviation. SPSS 19.0 software was applied to perform statistical analysis (IBM, Armonk, NY, USA), with analysis of variance. Pairwise comparisons (seast significant difference tests) between the groups were also conducted. $\mathrm{P}<0.05$ was considered to indicate a statistically significant difference.

\section{Results}

Lycopene significantly inhibits neointimal hyperplasia in restenosis models. To assess the effects of lycopene on neointimal hyperplasia, the changes in the IT/MT thickness ratio and the foam cell formation were determined by $\mathrm{H} \& \mathrm{E}$ staining and TEM in the restenosis models following drug administration. $\mathrm{H} \& \mathrm{E}$ staining demonstrated that the balloon injury and high-fat diet resulted in marked carotid intimal thickening (Fig. 1A), which confirmed the establishment of the rabbit restenosis models. Compared with the sham group, the IT/MT ratio in the carotid plaques in the model group was markedly elevated $(\mathrm{P}<0.01$; Fig. 1B). Apocynin treatment significantly reduced the IT/MT ratios in the carotid plaques in these models, which was further decreased by lycopene administration ( $\mathrm{P}<0.01$; Fig. 1B).

Furthermore, there were a large number of foam cells within the intima in the model and the apocynin-treated groups, whereas less foam cells were observed in the lycopene group. This finding was further confirmed by the TEM results. In the sham group, the smooth muscle cells exhibited contracting morphologies, with an abundance of intracellular actin fibers (Fig. 2). By contrast, in the model group, the actin fibers were markedly diminished within the smooth muscle cells, which were transformed into secreting cells. These cells contained a variety of types of cellular matrix and numerous large lipid droplets, exhibiting the phenotypes of smooth muscle cell-derived macrophages. However, the changes in the models were evidently alleviated by lycopene treatment (Fig. 2). The 

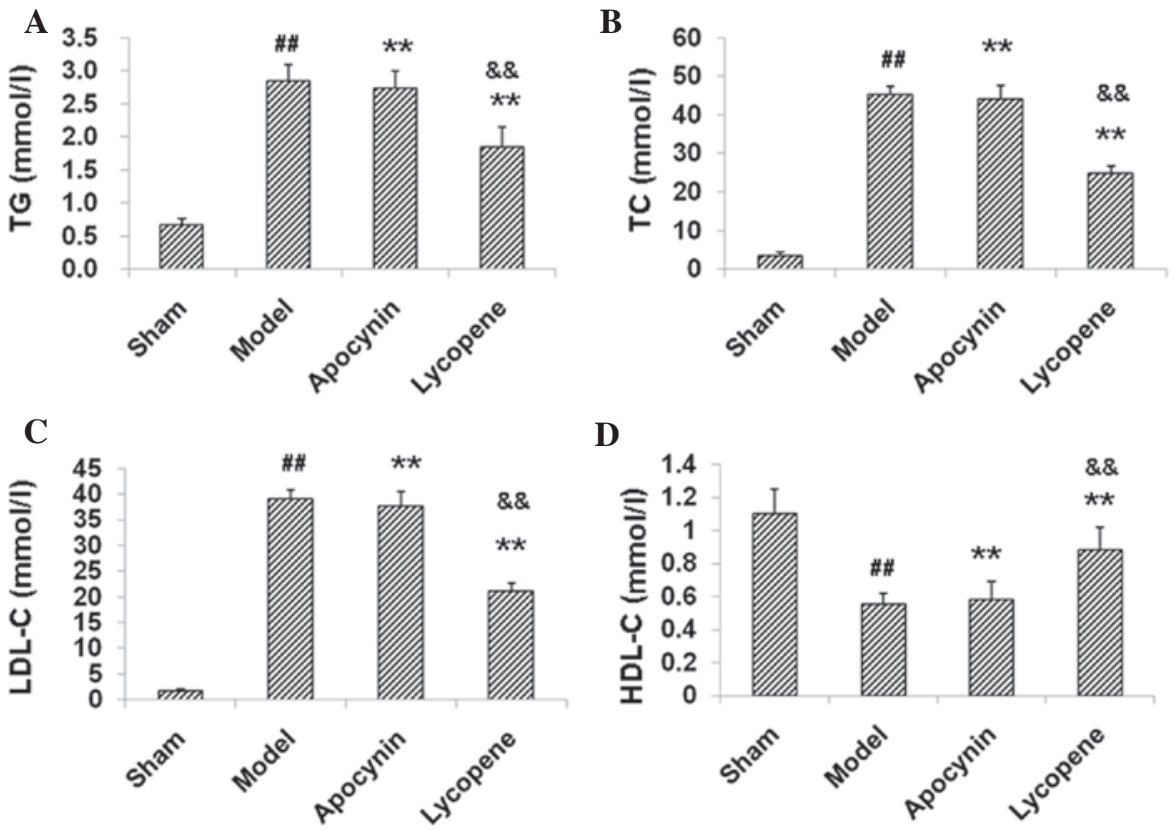

D

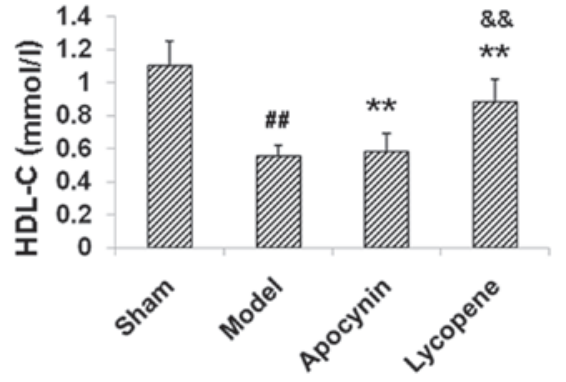

Figure 3. Lipid metabolism assessment in the restenosis models. Blood lipid levels in the rabbit restenosis models were measured, including (A) TG, (B) TC, (C) LDL-C and (D) HDL-C. Data are presented as the mean \pm standard deviation $(\mathrm{n}=8)$. ${ }^{\# \#} \mathrm{P}<0.01$, compared with the sham group; ${ }^{* *} \mathrm{P}<0.01$, compared with the model group; \&\& $\mathrm{P}<0.01$, compared with the apocynin group. TC, total cholesterol; TG, triglycerides; LDL-C, low-density lipoprotein cholesterol; HDL-C, high-density lipoprotein cholesterol.
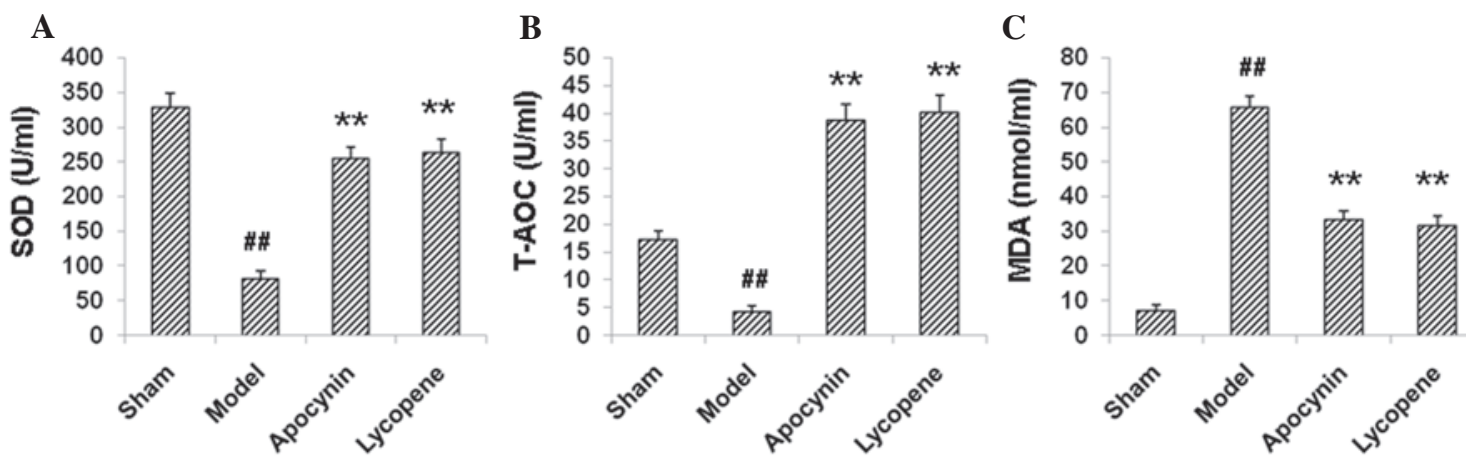

Figure 4. Oxidative stress detection in the restenosis models. Oxidative stress-associated parameters in the rabbit restenosis models were measured, including serum (A) SOD, (B) T-AOC and (C) MDA levels. Data are presented as the mean \pm standard deviation $(\mathrm{n}=8) .{ }^{* \prime \prime} \mathrm{P}<0.01$, compared with the sham group; ${ }^{* *} \mathrm{P}<0.01$, compared with the model group. SOD, superoxide dismutase; T-AOC, total anti-oxidant capacity; MDA, malondialdehyde.

above results suggested that lycopene significantly reduced the IT/MT ratios, the accumulation of lipids and the formation of foam cells, i.e., reducing neointimal hyperplasia, in the rabbit restenosis models.

Lycopene regulates blood lipid levels in restenosis models. As it was found that lycopene reduced the lipid accumulation and foam cell formation in restenosis models, the effects of lycopene on the lipid metabolism in these models were assessed. The blood lipid levels were measured and the results indicated that the levels of TC, TG and LDL-C were markedly increased, while HDL-C was decreased in the model group as compared with the sham group $(\mathrm{P}<0.01$; Fig. 3$)$. When treated with lycopene, the TC, TG and LDL-C levels were significantly decreased, while the HDL-C levels were significantly elevated in these models $(\mathrm{P}<0.01$;Fig. 3 ). However, apocynin did not induce any significant responses in these indexes in the models
( $P>0.05)$. These results suggested that lycopene regulated the blood lipid levels, which may be responsible for its therapeutic effects in the restenosis models.

Lycopene suppresses oxidative stress in restenosis models. The oxidative status has been closely linked with cell proliferative activities and lipid peroxidation. Therefore, the effects of lycopene on the oxidative status in the restenosis models were examined. The results demonstrated that, compared with the sham group, the levels of SOD and T-AOC significantly declined, and the contents of MDA were significantly elevated in the model group $(\mathrm{P}<0.01$; Fig. 4). Treatment with lycopene and apocynin upregulated SOD and T-AOC levels, while downregulating MDA levels $(\mathrm{P}<0.01$; Fig. 4). These results suggested that lycopene was able to suppress oxidative stress in restenosis models, which may reduce lipid metabolism and contribute to the reduced neointimal hyperplasia. 

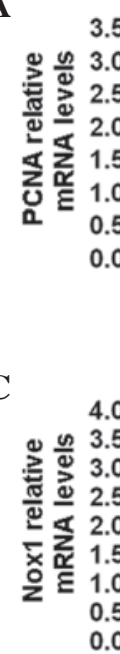

$\mathbf{E}$

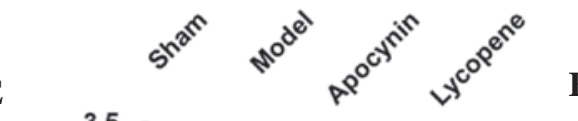

3.5

$-$

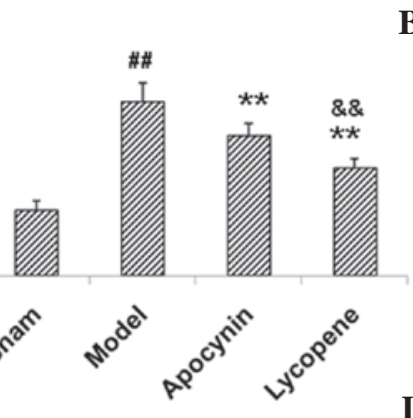

B

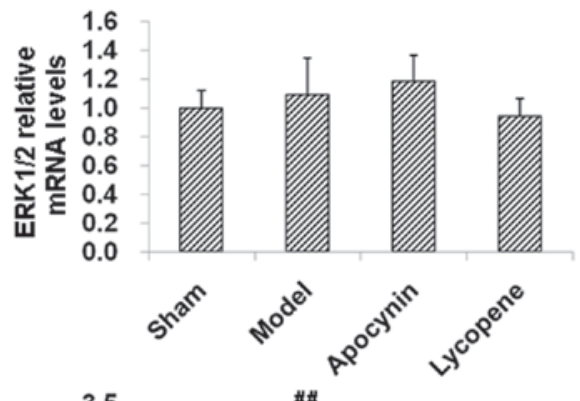

D 3.5

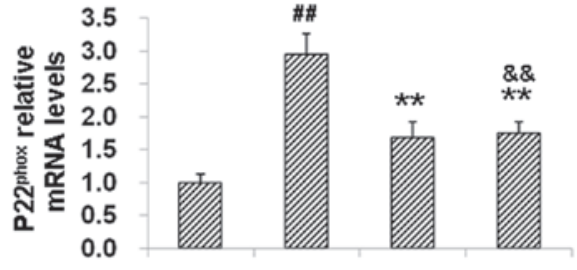

F
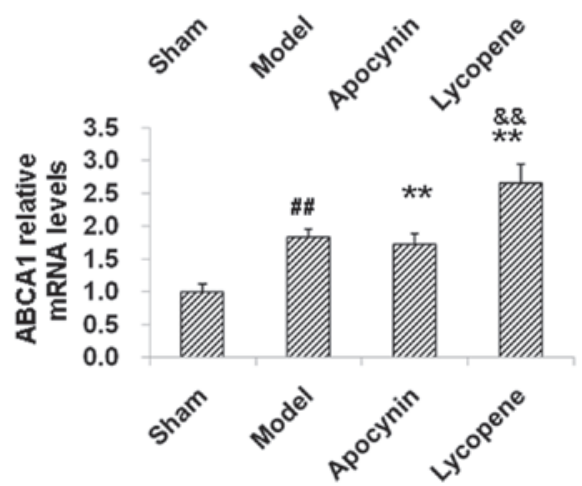

Figure 5. qPCR analysis of the levels of mRNAs involved in cell proliferation, oxidative stress and lipid metabolism in restenosis models. Expression levels of mRNAs involved in cell proliferation, oxidative stress and lipid metabolism in the restenosis models were detected by qPCR, including (A) PCNA, (B) ERK1/2, (C) Nox1, (D) p22 ${ }^{\text {phox }}$, (E) HMG-CoA reductase and (F) ABCA1. ${ }^{\#} \mathrm{P}<0.01$, compared with the sham group; ${ }^{* *} \mathrm{P}<0.01$, compared with the model group; ${ }^{\& \&} \mathrm{P}<0.01$, compared with the apocynin group. qPCR, quantitative PCR; PCNA; anti-proliferating cell nuclear antigen; ERK; extracellular signal-regulated kinase; Nox1, nicotinamide adenine dinucleotide phosphate oxidase 1; HMG-CoA R, hydroxymethyl glutaric acyl coenzyme A reductase; ABCA1, adenosine triphosphate-binding cassette transporter A1; $22^{\text {phox }}$, human neutrophil cytochrome $\mathrm{b}$ light chain.

Lycopene regulates the expression levels of proteins involved in cell proliferation and oxidative stress in restenosis models. To further investigate the mechanism(s) through which lycopene exerts its neointimal hyperplasia-inhibiting effects in restenosis models, the mRNA and protein expression levels of certain proteins involved in cell proliferation, oxidative stress and lipid metabolism were detected in the restenosis models using qPCR and western blot analysis. PCNA and p-ERK1/2 participate in the proliferation of cells. The results revealed that the mRNA expression levels of PCNA were elevated in the model group compared with the sham group, as indicated by qPCR (Fig. 5A). However, the PCNA mRNA levels in the models were significantly decreased by apocynin $(\mathrm{P}<0.01$; Fig. 5A). Compared with the apocynin group, the PCNA mRNA expression levels were further reduced by the treatment of lycopene $(\mathrm{P}<0.05$; Fig. 5A). Consistently, western blot analysis indicated that the elevated expression of PCNA in the restenosis models was reduced by lycopene and apocynin, with lycopene inducing a more potent reduction effect (Fig. 6A and B). There were no significant changes observed in the expression of ERK1/2 between these groups, neither at the mRNA (Fig. 5B) nor the protein (Fig. 6A) level. However, the expression levels of p-ERK1/2 were increased in the model group. Apocynin treatment significantly decreased the expression levels of p-ERK1/2 $(\mathrm{P}<0.01)$, which was further reduced by the administration of lycopene $(\mathrm{P}<0.05$, compared with the apocynin group; Fig. 6A and $\mathrm{C})$. These results suggested that lycopene significantly decreased the expression levels of cell proliferation-associated proteins.

Noxl and p22 $22^{\text {phox }}$ are associated with the cellular oxidative status. The results indicated that the expression of Nox1 and $\mathrm{p} 22^{\text {phox }}$ were markedly increased by restenosis at the mRNA (Fig. 5C and D) and protein (Fig. 6A, D and E) levels. Following the drug treatments, the expression levels of Nox1 and $\mathrm{p} 22^{\text {phox }}$ were decreased, confirming the anti-oxidant effects of lycopene and apocynin (Fig. 5C and D; Fig. 6A, $\mathrm{D}$ and $\mathrm{E})$. HMG-CoA reductase and ABCA1 are involved in cholesterol biosynthesis and efflux. The results from the qPCR and western blot analysis indicated that, in the model group, the mRNA and protein expression levels of HMG-CoA reductase and ABCA1 were elevated compared with the sham group (Fig. 5E and F). HMG-CoA reductase levels were decreased, while ABCA1 was further increased, both on the mRNA and protein expression level, following administration of lycopene $(\mathrm{P}<0.01)$. Apocynin, however, did not affect the expression levels of $\mathrm{HMG}-\mathrm{CoA}$ reductase and ABCA1 (Fig. 5E and F; Fig. 6A, F and G). Of note, there were 


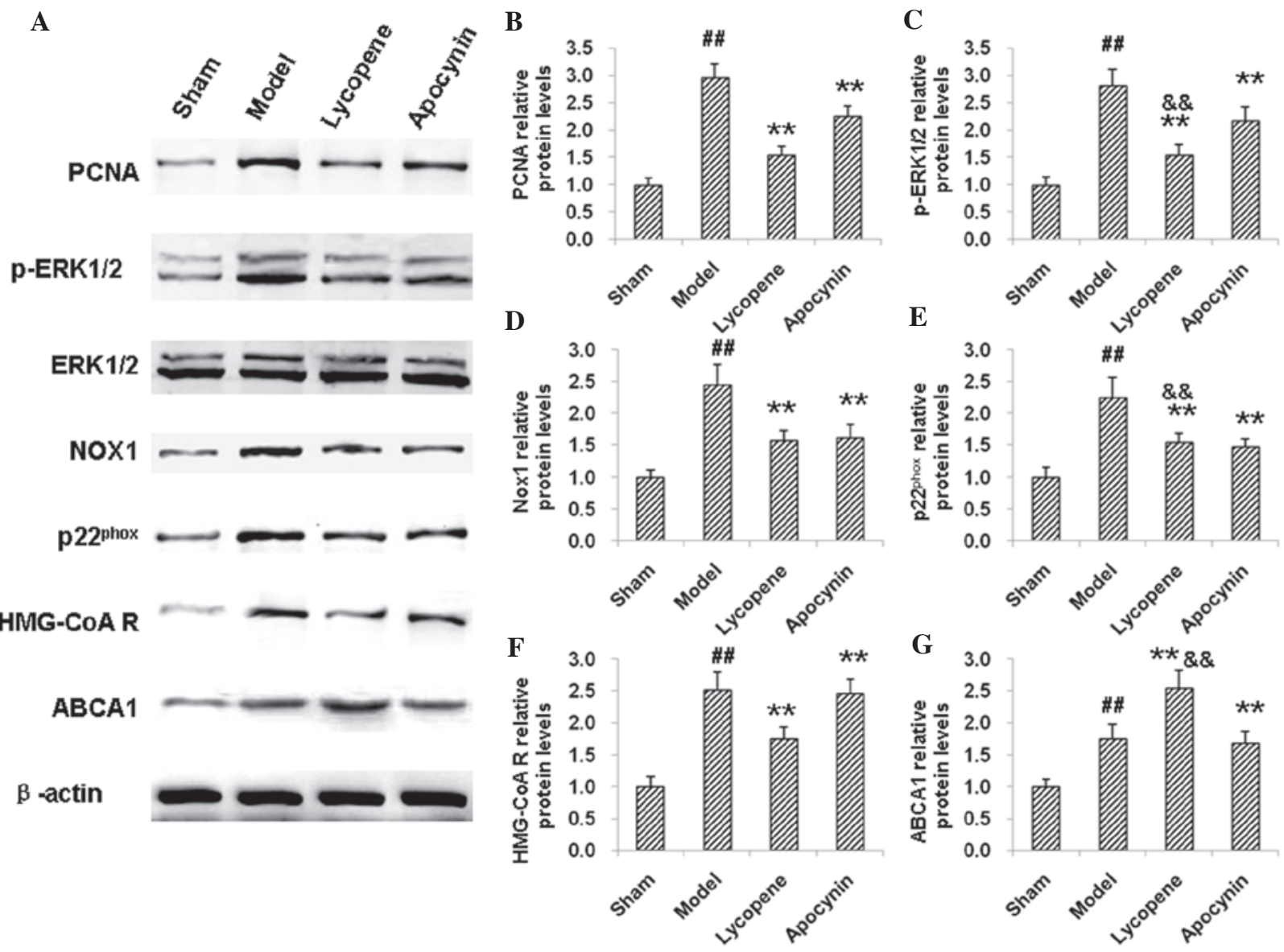

Figure 6. Western blot analysis of the expression levels of proteins involved in cell proliferation, oxidative stress and lipid metabolism in restenosis models. (A) Protein expression levels of proteins involved in cell proliferation, oxidative stress and lipid metabolism in restenosis models were detected by western blot analysis. Quantitative analysis of the expression levels of (B) PCNA, (C) p-ERK1/2, (D) Nox1, (E) p22 phox , (F) HMG-CoA reductase and (G) ABCA1. ${ }^{\# \#} \mathrm{P}<0.01$, compared with the sham group; ${ }^{* *} \mathrm{P}<0.01$, compared with the model group; \&\& $\mathrm{P}<0.01$, compared with the apocynin group. PCNA; anti-proliferating cell nuclear antigen; ERK; extracellular signal-regulated kinase; Nox1, nicotinamide adenine dinucleotide phosphate oxidase 1; HMG-CoA R, hydroxymethyl glutaric acyl coenzyme A reductase; ABCA1, adenosine triphosphate-binding cassette transporter A1; p22 ${ }^{\text {phox }}$, human neutrophil cytochrome b light chain.

significant differences in the mRNA and protein expression levels of HMG-CoA reductase and ABCA1 between the apocynin and the lycopene groups $(\mathrm{P}<0.05$ or $\mathrm{P}<0.01)$. These results suggest that lycopene regulates the processes of cholesterol biosynthesis and efflux, which may contribute to the inhibition of foam cell formation.

\section{Discussion}

Lycopene has strong antioxidant properties (12) and apocynin is a specific inhibitor of NADPH oxidase (13-15). These two compounds have been demonstrated to have numerous beneficial effects in cardiovascular diseases, particularly intimal hyperplasia. The present study investigated for the first time, to the best of our knowledge, the possible mechanisms through which lycopene inhibits neointimal hyperplasia. The results indicate that the inhibitory effects of lycopene on neointimal hyperplasia and foam cell formation are notably more potent than those of apocynin. In the rabbit restenosis models, lycopene exerted antioxidant effects, inhibiting the mRNA and protein expression of Nox 1 and $\mathrm{p} 22^{\text {phox }}$. In addition, lycopene regulated the expression of HMG-CoA reductase and ABCA1, which are involved in lipid metabolism. These activities may contribute to the neointimal hyperplasia-inhibiting effects of lycopene.

PCNA is a polypeptide only synthesized and expressed in the nuclei of proliferating cells, which may be used as an indicator for the assessment of cell proliferation $(3,16)$. As one of the most established mitogen-activated protein kinases, ERK1/2 have a key role in important cellular signaling pathways, mediating growth factor- and/or cytokine-associated cell proliferation (4). In the present study, the expression levels of PCNA and p-ERK1/2 were significantly reduced following lycopene administration in restenosis models, indicating a decreased cell proliferative activity, consistent with the inhibition of neointimal hyperplasia.

When the production of ROS exceeds its clearance, ROS is gradually accumulated inside cells and/or the organisms, causing oxidative stress. It has been suggested that the oxidative status affects the occurrence and development of neointimal proliferation (17-19). In addition, ROS are important signaling molecules in regulating the vascular functional status. ROS generated by NADPH oxidase serve as secondary messengers in the modulation of cell proliferation, differentiation and apoptosis, rather than providing cell defense functions (20). NADPH oxidase is a multiple protein 
complex composed of the catalytic subunit gp91 phox (also known as Nox2, located in the cell membrane), the regulatory subunit $\mathrm{p} 22^{\text {phox }}$, as well as other regulatory subunits in the cytoplasm. A series of NADPH oxidase catalytic subunits have been identified in various types of cells, including Nox1. Nox1, which is also a homologue of gp91 phox induces ROS to promote the proliferation of vascular smooth muscle cells (21-24). The SOD and T-AOC levels reflect the body's ability to eliminate oxygen free radicals (25). By contrast, HMG-CoA reductase is the rate-limiting enzyme in cholesterol synthesis and ABCA1 appears to have a key role in reverse cholesterol efflux and HDL generation (26-28). The results of the present study demonstrated that the expression of Nox 1 and $\mathrm{p} 22^{\text {phox }}$ were significantly elevated, while the SOD and T-AOC levels were significantly decreased in the restenosis models. Furthermore, the proteins involved in lipid metabolism, including HMG-CoA reductase and ABCA1, were also increased in these models. Lycopene treatment significantly downregulated the expression of Nox 1, p2 $2^{\text {phox }}$ and HNG-CoA reductases. However, the levels of SOD and T-AOC were upregulated, as well as the expression of ABCA1, in these models. These results provide molecular evidence for the lipid metabolism regulatory effects of lycopene.

In conclusion, both lycopene and apocynin significantly inhibit neointimal hyperplasia in the restenosis models caused by balloon injury, with lycopene treatment having a more potent effect. Lycopene exerts antioxidant functions, regulates plasma lipid levels and modulates the expression of mRNA and proteins involved in cell proliferation, oxidative stress and lipid metabolism, while apocynin generates limited effects in these restenosis models. The results suggested that lycopene suppresses oxidative stress and regulates lipid metabolism, representing a promising future therapeutic strategy against neointimal hyperplasia.

\section{Acknowledgements}

The present study was supported by the National Natural Science Foundation of China (nos. 30670869 and 30772295; key program, no. 30530360), the National Basic Research Program of China (973 program, nos. 2006CB503907 and 2008CB517309) and the Natural Science Foundation Project of CQ CSTC (no. 2008BA5016).

\section{References}

1. Model LS and Dardik A: Neointimal hyperplasia: Basic considerations. In Haimovici's Vascular Surgery. Ascher E (ed). 6th edition. Wiley-Blackwell, West Sussex, England, pp178-196, 2012.

2. Gao C, Xu W, Xiao W, Yu J and Li M: Simvastatin decreases stent-induced in-stent restenosis rate via downregulating the expression of PCNA and upregulating that of p27kip1. J Interv Cardiol 26: 384-391, 2013.

3. Mailand N, Gibbs-Seymour I and Bekker-Jensen S: Regulation of PCNA-protein interactions for genome stability. Nat Rev Mol Cell Biol 14: 269-282, 2013.

4. Lawan A, Shi H, Gatzke F and Bennett AM: Diversity and specificity of the mitogen-activated protein kinase phosphatase-1 functions. Cell Mol Life Sci 70: 223-237, 2013.
5. Carmeliet P: Mechanisms of angiogenesis and arteriogenesis, Nat Med 6: 389-395, 2000.

6. Chan EC, Datla SR, Dilley R, Hickey H, Drummond GR and Dusting GJ: Adventitial application of the NADPH oxidase inhibitor apocynin in vivo reduces neointima formation and endothelial dysfunction in rabbits. Cardiovasc Res 75: 710-718, 2007.

7. Wang L, Gaziano JM, Norkus EP, Buring JE and Sesso HD: Associations of plasma carotenoids with risk factors and biomarkers related to cardiovascular disease in middle-aged and older women. Am J Clin Nutr 88: 747-754, 2008.

8. Riccioni G, D'Orazio N, Palumbo N, Bucciarelli V, Ilio ED, Bazzano LA and Bucciarelli T: Relationship between plasma antioxidant concentrations and carotid intima-media thickness: the Asymptomatic Carotid Atherosclerotic Disease In Manfredonia Study. Eur J Cardiovasc Prev Rehabil 16: 351-357, 2009.

9. Hozawa A, Jacobs DR Jr, Steffes MW, Gross MD, Steffen LM and Lee DH: Relationships of circulating carotenoid concentrations with several markers of inflammation, oxidative stress, and endothelial dysfunction: the Coronary Artery Risk Development in Young Adults (CARDIA)/Young Adult Longitudinal Trends in Antioxidants (YALTA) study. Clin Chem 53: 447-455, 2007.

10. Sesso HD: Carotenoids and cardiovascular disease: what research gaps remain? Curr Opin Lipidol 17: 11-16, 2006.

11. Rao AV, Ray MR and Rao LG: Lycopene. Adv Food Nutr Res 51: 99-164, 2006.

12. Palozza P, Catalano A, Simone R and Cittadini A: Lycopene as a guardian of redox signalling. Acta Biochim Pol 59: 21-25, 2012.

13. Stefanska J and Pawliczak R: Apocynin: molecular aptitudes. Mediators Inflamm 2008: 106507, 2008

14. Yu J, Weïwer M, Linhardt RJ and Dordick JS: The role of the methoxyphenol apocynin, a vascular NADPH oxidase inhibitor, as a chemopreventative agent in the potential treatment of cardiovascular diseases. Curr Vasc Pharmacol 6: 204-217, 2008

15. Kleniewska P, Piechota A, Skibska B and Gorąca A: The NADPH oxidase family and its inhibitors. Arch Immunol Ther Exp (Warsz) 60: 277-294, 2012.

16. Marx SO, Totary-Jain H and Marks AR: Vascular smooth muscle cell proliferation in restenosis. Circ Cardiovasc Interv 4: 104-111, 2011.

17. Zhang J, Chen J, Yang J, Xu CW, Pu P, Ding JW and Jiang H: Resveratrol attenuates oxidative stress induced by balloon injury in the rat carotid artery through actions on the ERK $1 / 2$ and NF-kappa B pathway. Cell Physiol Biochem 31: 230-241, 2013.

18. Antoniades C: Oxidative stress in the vascular wall: a useful physiological process or a therapeutic target in vascular disease? Recent Pat Cardiovasc Drug Discov 6: 74-77, 2011.

19. Li H, Horke S and Förstermann U: Oxidative stress in vascular disease and its pharmacological prevention. Trends Pharmacol Sci 34: 313-319, 2013.

20. Takac I, Schröder K and Brandes RP: The Nox family of NADPH oxidases: friend or foe of the vascular system? Curr Hypertens Rep 14: 70-78, 2012.

21. Yin W: The role and regulatory mechanisms of nox 1 in vascular systems. 2012.

22. Yin W and Voit EO: Function and design of the Nox1 system in vascular smooth muscle cells. BMC Syst Biol 7: 1-20, 2013.

23. Lee MY, San Martin A, Mehta PK, et al: Mechanisms of vascular smooth muscle NADPH oxidase 1 (Nox1) contribution to injury-induced neointimal formation. Arterioscler Thromb Vasc Biol 29: 480-487, 2009.

24. Ellmark SH, Dusting GJ, Fui MN, Guzzo-Pernell N and Drummond GR: The contribution of Nox4 to NADPH oxidase activity in mouse vascular smooth muscle. Cardiovasc Res 65: 495-504, 2005.

25. Liu DH, Chen YM, Liu Y, et al: Rb1 protects endothelial cells from hydrogen peroxide-induced cell senescence by modulating redox status. Biol Pharm Bull 34: 1072-1077, 2011.

26. Francone OL and Aiello RJ: ABCA1: regulation, function and relationship to atherosclerosis. Curr Opin Investig Drugs 3: 415-419, 2002.

27. Soumian S, Albrecht C, Davies AH and Gibbs RG: ABCA1 and atherosclerosis. Vasc Med 10: 109-119, 2005.

28. Attie AD: ABCA1: at the nexus of cholesterol, HDL and atherosclerosis. Trends Biochem Sci 32: 172-179, 2007. 